\title{
FIRST TESTS WITH FULLY DEPLETED PN-CCD'S
}

\author{
L. Strüder, G. Lutz, M. Sterzik
}

\author{
Max-Planck-Institut für Physik und Astrophysik \\ Werner-Ileisenberg-Institut für Physik \\ Föhringer Ring 6, D-8000 Mlünchen 40, FRG \\ P. Holl, J. Kemmer, U. Prechtel, T. Ziemann \\ Messerschmitt-Bölkow-Blohm GmbH \\ Postiach $8011 \div 9$, s000 München 80, FRG \\ P. Rehak \\ Brookhaven National Laboratory \\ Upton, N.Y. 11973 USA \\ BNL --40874 \\ DE88 007573
}

\section{Nowrace}

We have fabricated $280 \mathrm{~mm}$ thick fully depletable pn CCD's on high resistivity silicon $(g \approx 2.5 \mathrm{k} \Omega \mathrm{cm})$. Its operation is based on the semiconductor drift chamber principle proposed by Gatti and Rehak. They are designed as energy and position sensitive radiation detectors for (minimum) ionizing particles and $X$. ray imaging. Two dimensional semiconductor device modeliny demonstrates the basic charge transfer mechanisms. Prototypes of the detcctors have been tested in static and dynamic conditions. A preliminary charge transfer inelliciency was deternimed to $6 \cdot 10^{-3}$. The charge loss during the transfer is discussed and as a consequence we have developed an improved design for a second fabrication itcration which is now being produced.

\section{Introduction}

The implementation of comnercially available buried channel charge coupled levices (BCCD's) in the NA32 tixed target experiment at the SPS at CER:Y lias lead to a significant improvement of vertex reconstruction necded for the precise lifetine measurement of charmed particles [2]. The advantages of the (CD's have been a good two track resolution of $40 \mathrm{~mm}$ al. lowing to install them close to the interaction region and the good two dimensional position accuracy of $5 \mu \mathrm{m}$ in both dimensions rejecting a large fracticn of the combinatorial background from the one dimensional position information of the silicon strip vertex detector.

But the use of the new components has also pointed to some limitations of standard MOS CCD's. Due to the shallow depletion depth of approximately 8 fun only 1000 to 1.500 electrons are collected in the pixels by drift and diffusion from the depleted and adjacent undepleted silicon. In order to reduce all thernal noise contributions the detertor and the front end electronics had to be cooled to ISO $K$. The second ntajor disadvantage for their use in high energy physics experiments arises directly from the device performance: Because the signal charges are transfreed only $\mathrm{l} \mu \mathrm{m}$ from the $\mathrm{SiO}_{2}-\mathrm{Si}$ interfare the system is very sensitive to radiation damage. Every $10^{1}$ Pad intrgrated dose of particles the voltage at the transfor gatos has to be increased about $l V$ to compensate the additionally created fixed space charge in the silicon oxide.

The new concept of the presented (CD orctomes these restrictiong by increasing the scnsitive deptly of the sensor to $280 \mu \mathrm{m}$ resulting in a total of 20.000 to 30.000 signal electrons and in avoiding MOS structures. Because the entire semiconductor is drained of mobile charge carriers we call the device fully depleted CCD and because all the potentials in the semiconductor are steered and defined by pn junctions instead of MOS structures we call it according to its topology pn CCD. In ad. dition to the above mentioned properties the pn CCD will have a fast clear mechanism [3] by forward biasing the $\mathrm{p}^{+}$electrodes storing the signal charges. By thermionic emission processes we inject holes from the electrodes in a controlled way which recombine with the signal charges.

For the theasurement of low momenturn tracks multiple scattering in the silicon of the particles under study represents a serious limitation. A fully depleted detector, thus fully sensitive over the entire volume represents the optimum performance (best signal to noise ratio for a given detector thickness) with regard to the position uncertainty due to multiple scattering.

We first present the basic ideas of the fully depleted pn CCD's derived from the seniconductor drift clamber principle presented by Gatti and Reliak [1]. Two dimensional device simulations give a clear idea of the electrical behaviour dependent of the physical, geometrical and technological input data.

The presentation of the tests will be introdured by currentvoltage measurements to determine the semiconductor properties of the devices. The test of the dynamic behavtour of the pn $\mathrm{CCD}$ 's under typical operating contlitions proves the function principles of the devices of the lirst fabrication itcration. Limitations resulting from the non optimum lirst design will be discussed.

\section{Fully Depleted pn C('D)'s}

In 198:3 Gatti and Rehak proposed a new detector scheme based on the idea that a large smiconductor wafer of e.g. high resistivity $\mathbf{n}$ - type silicon can he depleted from a small $n^{+}$ohnic rontact positively binsed with respect to the planar $p^{+}$contacts covering both surfaces of the wafer. The depletion zone with expand from both rectifying junctions simultancously as long as the ohmic access from the $n^{+}$anode to the entirc bulk is not interrupted. At a given voltage the depleted regions touch each other. Inder this condution the furmer conductive electron chan-

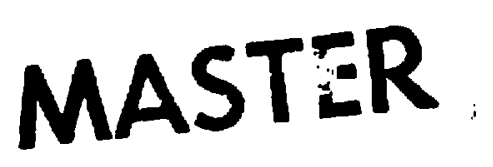

$\therefore 1$, 
nel symmetrically located in the middle of the substrate between the large $p^{+}$implants will abruptly disappear. At this moment the depletion is completed at a voltage which is four times lower than the voltage necded to deplete a simple diode of the same thickuess. The abowe described remowal of mobile charge carriers is called sideward depletiun. In this case the electron potcutial chergy itl a cul perpendicular to the wafer sutface has a parabolic shape. with an electron potential mininum in the middle of the water. The electron potential can be moved out of the center towards either surface by applying non equal voltages to the two $p^{+}$contacts.

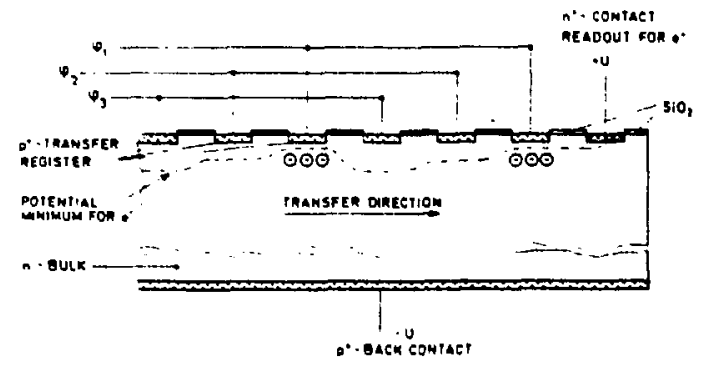

Fig. I: Basic configurntion of CCD topology

The advantinges of the technique are evident: Independently of the size of the depletion region the capacitance of the $n^{+}$ anude can be kept extremely small - necessary condition for good noise performance - and the position of the potential minimum can be controked by external means. This scheme is adapted for the operation of fully depleted pn CCD's. The basic topology is shown in Fig. 1. The $\mathrm{p}^{+}$back contact is negatively biased with respect to the shift electrodes on the top side thereby shifting the electron potential minitnum close top surface. By an appropriate choice of the toltages at the $p^{+}$tranfer registers $\psi_{1}, \neq 2$, and $>3$ it is possible to rreate local potential minima for clectrons and an adequate change with time in these voltages allows a discrete shift of the signal charges towards the readout anode.

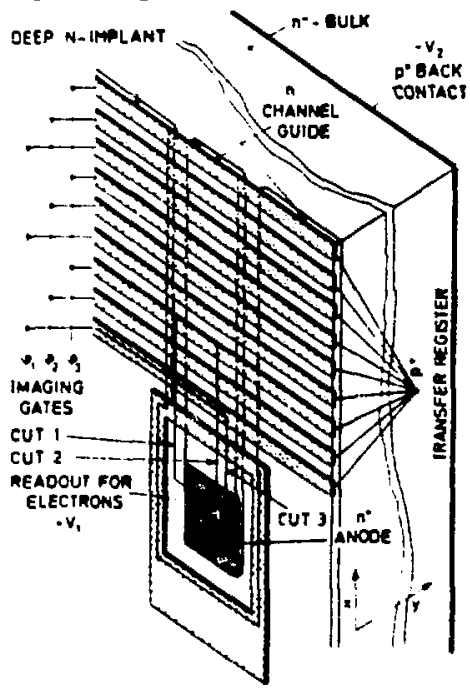

Fig 2: Srhemaluc trew of the linear (CD geometry with implandation topologies encluding channel stop mechanism
The more complex three dimensional arrangement of a linear CCD structure including the readout region with the $\mathrm{p}^{+}$guard ring is shown in Fig. 2. A deep $n$ - implantation below the $p^{+}$ transfer registers and below the $\mathrm{SiO}_{2}$ is introduced a) to prevent large liermally injected currents (reach through currents) between $p^{+}$junctions at different potentials and b) to form a guiding channel for signal charges. The lateral spread of the charges is prevented by the gap of the deep $n$ - implant between neighboring guiding channels. The absence of the positive space charge in the vicinity of a channel guide crcates a negative potential for electrons with respect to the depleted deep $n$-doped region, thus acting as a potential barrier for the signal charges, confining them to the channel guide area [3].

\section{Device Modeling}

We use device modeling as a powerful tool for feasibility studies of new semiconductor devices and for establishing design criteria for the mask layout. The interpretation of the putential and charge carrier distributions allows a detailed understanding of the electrical behaviour and a first estimation of the expected properties. In addition to it the related process simulation yields process parameters for the fabrication as implantation doses and -energies and diffusion- and annealing times.

The non-equilibrium potential distribution of one picture cell was calculated by solving the Poisson equation with a Fast Fourier Transform program. The inlluence of the fixed $\mathrm{SiO}_{2}$. $\mathrm{Si}$ interface charges and the inhomogenious doping close to the pixel structure are taken into account.

The steady state behaviour was studied with a two dimen. sional program solving Poisson-, Transport- and Continuity equation simultaneously by iterative means, describing the operation conditions of the CCD, when all pixels are completely filled up with signal charges.

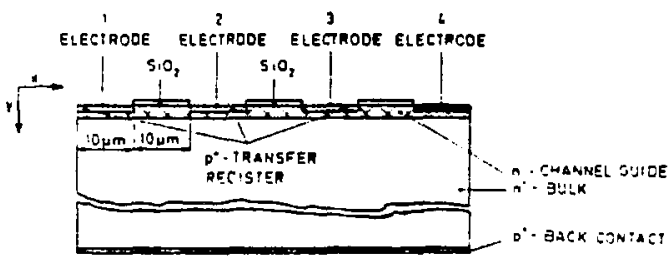

Fig. 3: Schematically drau'r geometry for the devee simulation corresponding to "cut is" in Fig. "?

Fig. 3 shows the geometrical arrangement and the implantation topology for the non equilibrium simulation.

The potential energy distribution of a three phase (CD) pixel under typical operation conditions is plotted in Fig. 1 . The potential applied at the 1 . electrode is $-10 \mathrm{~V}(r)$, it the 2 . electrote $-i V(+2)$ and at the 3 . electrode $-4 V(+3)$. The 4 . electrode corresponding to $p q$ once more is set in $-10 \downarrow$ The voltage at the backside - in this calculation locarrod at a drpth of $100 \mathrm{~km}$ - is equivalent in the real operation voltage of $-70 \mathrm{~V}$ in the $280 \mu \mathrm{m}$ thick fully depleted device.

its can be seen from the related equipotential plot in Fig. 5 the individual pixels are electrically separated from each uther. electrons accumulated under the $\mathrm{SiO}_{2}$ do not interiere wh the sigual charges and the potential ninimum for electrom 1 , lorated at a distance of approximately $35 \mu \mathrm{m}$ from the surfac iaving the pixel structure 


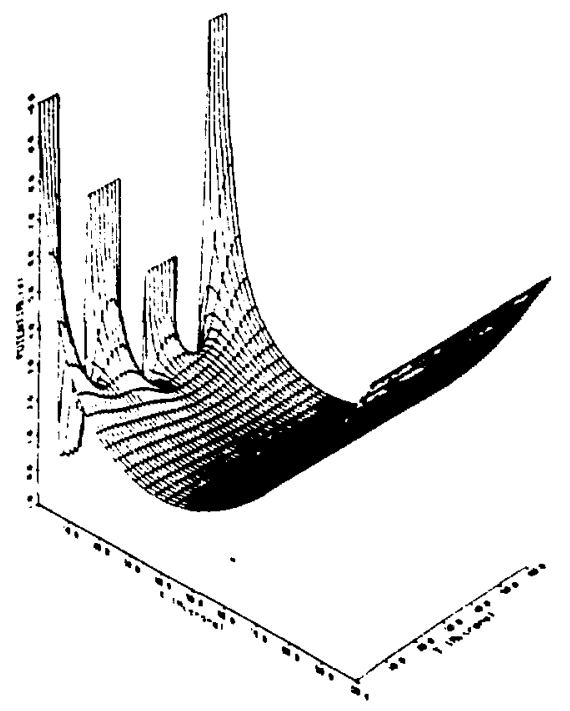

Fig. f: Potentral energy distribution of one pirel under non equilibrium conditions

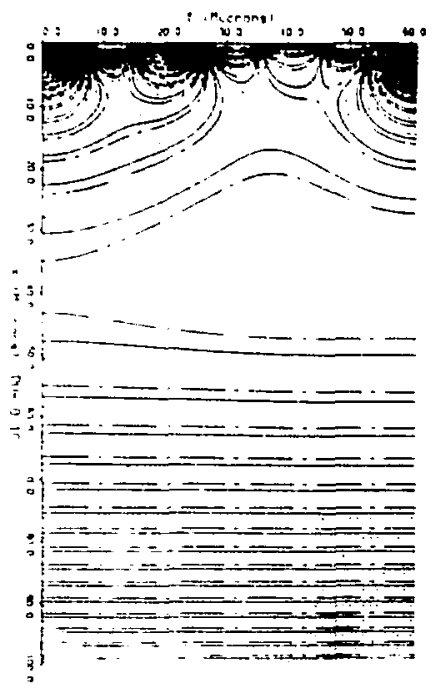

Fig. 5: Equipotential of a CCD pizel under non equilibrium ronditions

\section{First [ests of a Linear Fully Depeleted CCD}

The CCD measurements presented below refer to a lincar array of $3: 3$ pixels with a pixel size of $150 \times 100 \mu \mathrm{m}^{2}$

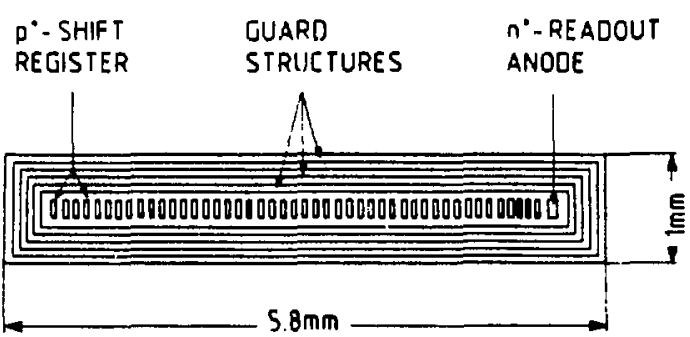

Fig. 6: Sketch of the device under test

A scheme of the device seen from the top is drawn in Fig. 6. Only the $\mathrm{p}^{+}$shift register and the $n^{+}$readout anode area have the previously introduced deep $\mathbf{n}$ - implantation as channel guide for the signal charges. The $\mathrm{p}^{+}$guard structures prevent the contamination of the signals from thermally generated charge carriers from the outer part of the chip. An appropriate negative voltage at these $\mathrm{p}^{+}$junctions also focuses the signal charges towards the sensitive channel guide while drifting and diffusing across the wafer.

\section{Static measurements}

Numerous current-voltage (I/V) and capacity-voltage (C/V) measurements have been performed to provide informations about the fabrication process, the device characteristics and the criteria for device selection such as confirmation of the expected depletion characteristics, low leakage currents at full depletion and long term stability.

Three examples are shown in Fig. 7 - 9 .

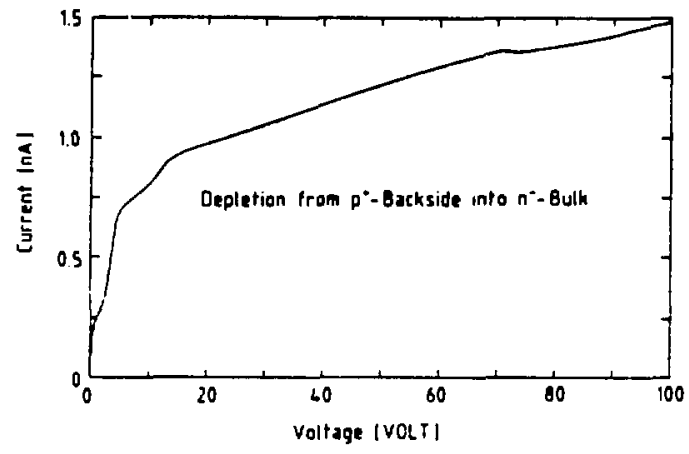

Fig. 7: Leaknge current arising from bulk depietion

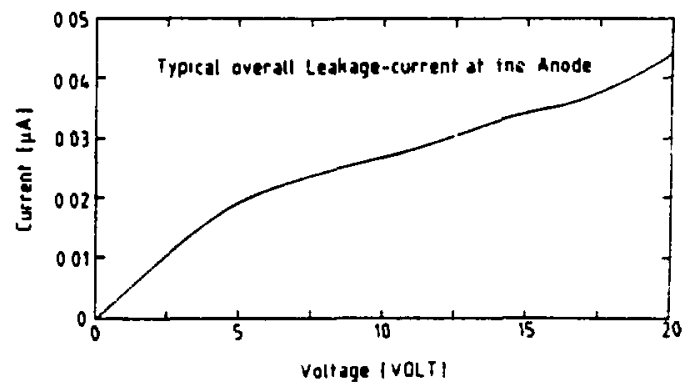

Fig. 8: Leakage current collected at the ${ }^{+}$anoile as functhon of the voltage of the $p^{+}$clectrondes on the top side. Complete depletion frain the backszde with a constunt potential at $.100 \mathrm{~V}$ 
Depleting the CCD from the $p^{+}$backside the cursent col. lecterl at the anute is mainly due to generation and recombina. tion in the bulk and to injection of minority carriers from the $\mathrm{p}^{+}$junction (Fig. $i$ ). Note that the deep $n$ - implantation under the $\mathrm{p}^{+}$transfer register and the volume directly under the $\mathrm{SiO}_{2}$ is not yet depleted.

In order to measure the entite leakage curtent contribution lumiting the resolution of the CCD output signal the bulk is depleted with a constant bias at the back contact of $-100 \mathrm{~V}$. The $\mathrm{A}^{+}$anude is reserse biased with respect to all $\mathrm{p}^{+}$conlacts on

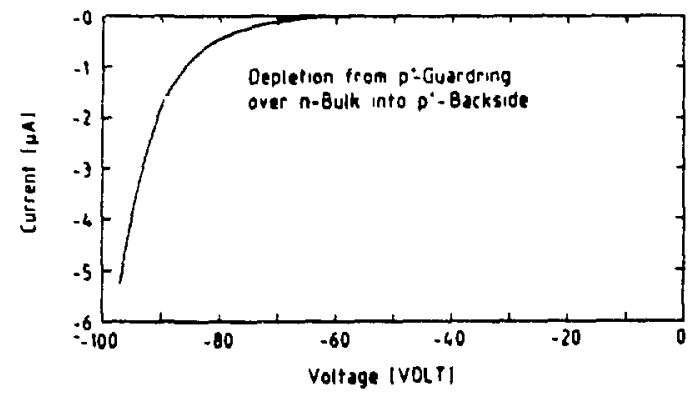

Fig. 9: liench through current characicristic of a $p^{+} n^{-} p^{+}$ structure formed by the $p^{+}$guard rings and the $p^{+}$backcontact and the high resistivity n - type bulk

the top side leading to the $/ / \mathrm{H}$ characteristic shown in Fig. 8 displaying the overall current collected at the readout node.

Fig. 9 gives the hole current thermionica:.y injected from the $p^{+}$guard structures from the top side through the $n^{-}$bulk into the $p^{+}$back contact. The $p^{+}$guard structures have no underlying deep $n$ implant.

From this type of measurements snitable operation conditions can be extracted.

$$
\begin{aligned}
V_{\text {back }} & =-10 \mathrm{~V} \\
V_{\text {anoite }} & =+10 \mathrm{~V} \\
\forall_{\text {shzst }} & =+3 \mathrm{~V} \rightarrow-3 \mathrm{~V} \\
V_{\text {guard }} & =-25 \mathrm{~V}
\end{aligned}
$$

C $/ \mathrm{l}$ measuremeats confirm full depletion under these condutions.

\section{Dytamic measurements}

In our experimental setup the signal of ionizing particles is sumulated will a pulsed infrared laser illuminaled from the backstle $(\lambda=904 \mathrm{~nm})$ with an average penetration depth of about $20 \mu \mathrm{m}$. Each light pulse creates 250.000 electron hole pairs simulatung 10 minimum ionizing particles in a $290 \mu \mathrm{m}$ thick waler. The repetition tate was $\mathbf{5 0 0} \mathrm{Hz}$. The laser system also supplies a trigger signal for the readout while the $(* 1)$ is running continuusly under the above described operation conditions. The three shift registers are clocked with square wases at $1 / 6 \mathrm{MHz}$.

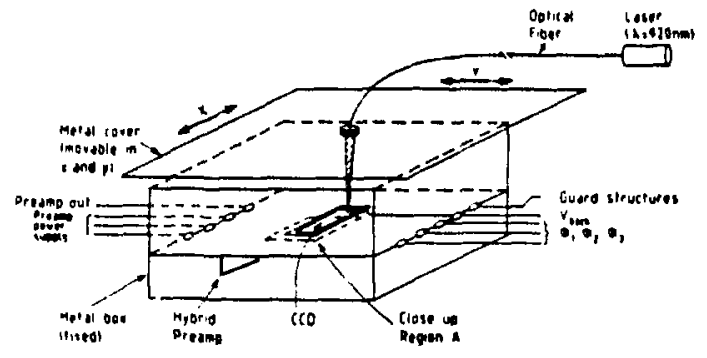

Fig. 10: Experimental setup

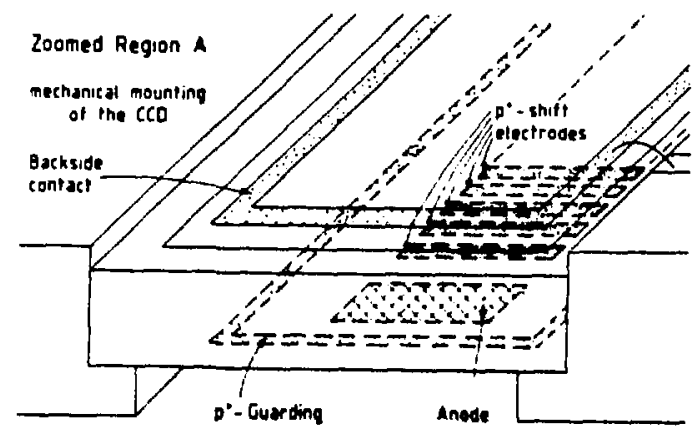

Fig. Il: Hounting of the chip on the printed circuit board

The (CD response was online monitored on a digitizing oscilloscope. The experimental setup and the mounting of the chip is shown in Fig. $10-11$. The four consecutive plots (Fig. 12 - 15) display the transfer of the injected signal charges to the anode: The variation of the position of the laser spot causes a correlated relay of the electron signal response reaching the readout anode at different times.

As the clock frequency is $167 \mathrm{kHz}$ the transfer time for one pixel is $6 \mu \mathrm{s}$. With a pixel width of $150 \mu \mathrm{m}$ the distance of 1.5 min ( $\equiv 10$ pixels) from the anode to the laser spot corresponds to a time delay of 60 as on the oscilloscope in Fig. 13. The significantly higher signal in Fig. 12 where the anode region is

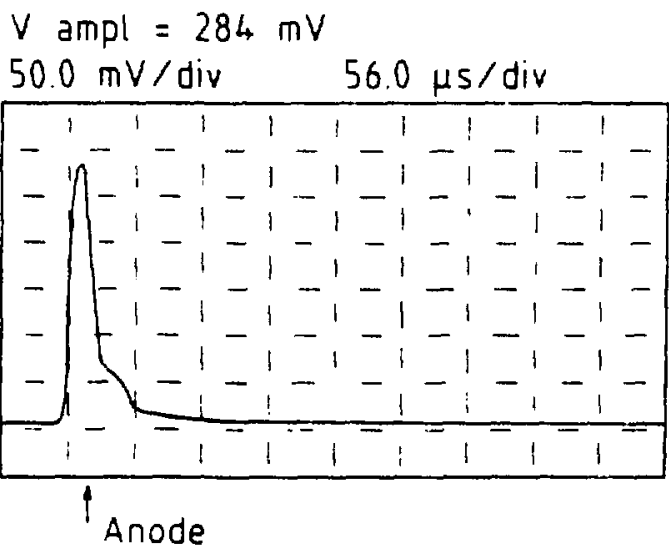

Fig. 12: Lawer spot injected from the buck sade in the anode region 
$V$ ampl $=155 \mathrm{mV}$

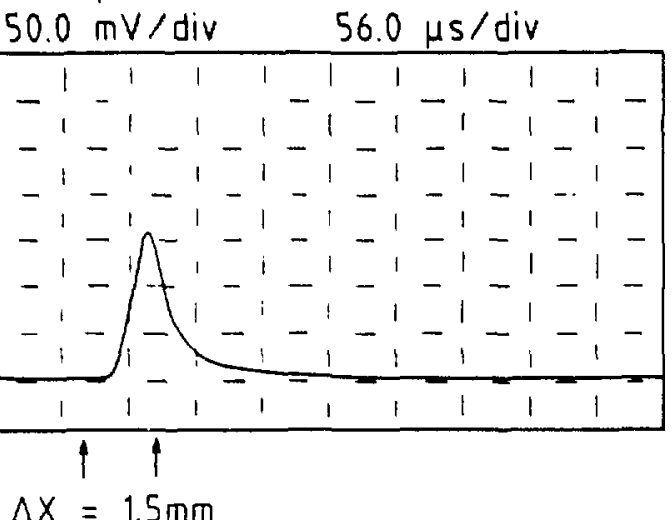

Fig. 13: Laser spot injected from the back side 10 pirels far from the anode

$V$ ampl $=148 \mathrm{mV}$

$50.0 \mathrm{mV} / \operatorname{div} \quad 56.0 \mu \mathrm{s} / \mathrm{div}$

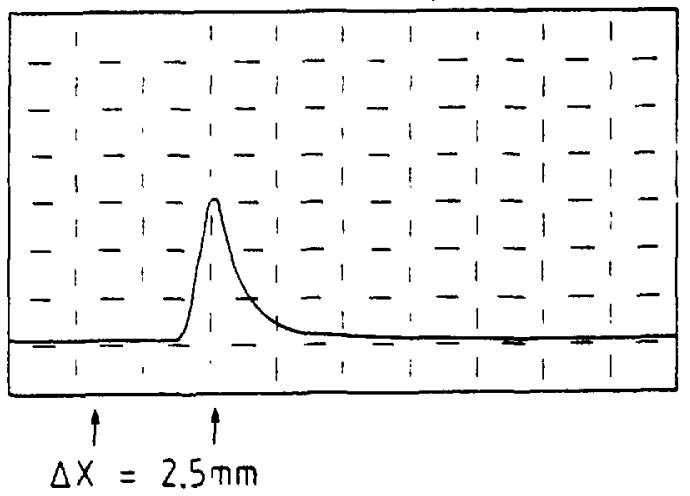

Fig. 1.f: Laser spoi injected from the back side 17 pixels for from the anode

$V$ ampl $=142 \mathrm{mV}$

$50.0 \mathrm{mV} /$ div $\quad 56.0 \mu \mathrm{s} / \mathrm{div}$

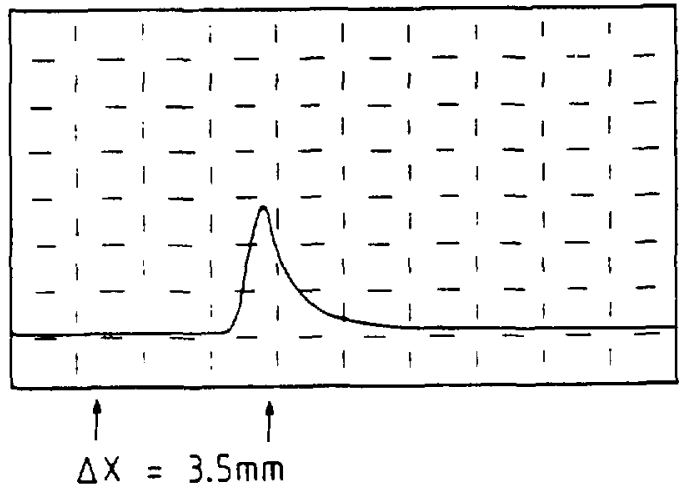

Fig. 1.5: Laser spot injected from the back stide 24 pizels far from the anode directly illuminated is due to the effective larger sensitive area around the $\mathrm{n}^{+}$readout contact completely containing the light spot. The width of the output pulse is mainly caused by the spot size of the laser (about $500 \mu \mathrm{m}$ ) and the shaping of the preamplifier signal. The decrease of the slope of the trailing edge in Fig. 13-15 can be attributed to a backward How loss of signal charges. From the decreasing pulse toight in Fig. 13-15 a preliminary transfer efficiency of 0.994 per pixe! was determined.

\section{Discussion and outlook}

The loss of signal charges can be mainly attributed to the weak electrical control of the $\mathrm{SiO}_{2}$ regions within the pixel structure. They seem to be short circuited due to the presence of the electron accumulation layer at the $\mathrm{SiO}_{2}-\mathrm{Si}$ interface. In order to reduce the mixture of signal charges with the accumulated electrons we have to displace the charge transfer deep in the bulk. But the control of the potentials at that distance becomes very ineffective resulting in small fringing fields for signal transport giving rise to increased backward flow losses.

An improved transfer efliciency is expected with a more suitable transfer gate structure which allows signal charge shifting closer to the surface. A new charge is being produced taking into account the experiences of the first tests. The inzegration of the preamplifier on the CCD for better noise performance is actually under study [4]. A first version of the circuit is realized on the same chip.

The confirmation of the function principles of fully depleted pn CCD's has lead to an extended feasibility analysis for X-ray detection in the X.MM satellite experiment.

\section{REFERENCES}

1. E. Gatti, P. Rehak: Semiconductor Drift Chamber - an Application of a Novel Charge Transport Scheme. in Proc. 2nd P'isa Meeting on Advanced Detectors, Grosetto. Italy(198.3) NIM A, Vol. 225 (198.1) pp.608.614.

2. Barlag, S. et al. Measurement of the Lifetime of the Charmed Baryon $\lambda_{e}$, CERN-EP/86-173, submitted to Physics Letters $B$

3. Development of Fully Depletable CCD's for Uigh Energy Physics Applications, NIM A, Vol. 25i (1987), pp. i394-602.

4. Design of Charge Preamplifiers on High Resistivity Detector Grade Silicon. Radeka. $V$. et al, contsjbution to the IEEE 1987 Nuclear Science Symposium. to be published in the conference proceedings

Th1s research supported in part by the U.S. Dept, of Energy under contract DE-AC02-76CH0OO16.

\section{DISCLAIMER}

This report was prepared as an account of work sponsored by an agency of the United States Government. Neither the United States Government nor any agency thereof, nor any of their employees, makes any warranty, express or implied, or assumes any legal liability or responsibility for the accuracy, completeness, or usefulness of any information, apparatus, product, or process disclosed, or represents that its use would not infringe privately owned rights. Reference herein to any specific commercial product, process, or service by trade name, trademark, manufacturer, or otherwise does not necessarily constitute or imply its endorsement, recommendation, or favoring by the United States Government or any agency thereof. The views mendation, or favoring by the Uned herejn do not necessarily slate or reflect those of the United States Government or any agency thereof. 